\title{
A 5-Fr Externalized Nephroureteral Catheter as the Sole Protective Device for Pediatric Pyeloplasty: The Experiences of 142 Patients
}

\author{
Mansour Mollaeian, ${ }^{1}$ Maryam Ghavami-Adel, ${ }^{1,}$ Farid Eskandari,, and Arash Mollaeian ${ }^{2}$ \\ ${ }^{1}$ Department of Pediatric Surgery, Tehran University of Medical Sciences, Tehran, IR Iran \\ ${ }^{2}$ General physician, Tehran, IR Iran \\ "Corresponding author: Maryam Ghavami-Adel, Department of Pediatric Surgery, Imam Khomeini Hospital Complex, Valiasr Hospital, Tehran, IR Iran. E-mail: \\ mghadel@yahoo.ca
}

Received 2016 February 06; Revised 2016 March 10; Accepted 2016 March 31.

\begin{abstract}
Background: Pyeloplasty for ureteropelvic junction obstruction correction is a common procedure, but the optimal method for protective diversion after pyeloplasty is still a matter of debate.

Objectives: Here, we present our clinical trial experience using a single percutaneous externalized nephroureteral (NU) 5-Fr catheter (infant feeding tube) with multiple side holes as the sole instrument of drainage to provide a protective mechanism.

Materials and Methods: In this prospective study, we analyzed the charts of 142 patients who underwent pyeloplasty from August 2001 through October 2008. We used a single externalized NU 5-Fr catheter with multiple side holes for postoperative upper tract diversion. The catheter was removed in the office after 10 - 14 days. Complications from the use of this catheter, including poor catheter function, premature dislodgement, urinary tract infection, leakage, urinoma, and anastomotic stenosis, were evaluated. The operations were performed by two surgeons at two separate centers.

Results: In all, 148 pyeloplasty procedures were performed on 142 patients. The mean hospital stay length was 2 (1-3) days. A contrast study through a catheter demonstrated excellent drainage with no leakage in all patients. Immediately after catheter removal, febrile urinary tract infection and transient obstructive symptoms and signs occurred in 15 patients.

Conclusions: Using a percutaneous externalized NU 5-Fr catheter was sufficient as a protective measure after open pyeloplasty. It costs less than other diverting systems, such as DJ, and can be removed in the office. Therefore, it can be a safe and cost effective procedure, especially in developing countries where cystoscopic set ups are not readily available. There were only a few notable complications.
\end{abstract}

Keywords: Pyeloplasty, UPJO, Diversion, Nephroureteral Catheter, Percutaneous Catheter

\section{Background}

Anastomotic leakage and protective urinary diversion in pediatric pyeloplasty continue to be a controversial issue among pediatric urologists $(1,2)$. Some surgeons prefer a tubeless repair, while others use some varied combination of instruments, including an internal ureteral stent, nephrostomy tube, or a nephron-ureteral stent (1) along with a perinephric drain and/or urethral bladder catheter $(3,4)$.

The pediatric literature reports a complication rate of $12 \%$ for the stented group and $14 \%$ for the un-stented group (4).

\section{Objectives}

In this article, we attempt to demonstrate that a single percutaneous externalized nephroureteral (NU) 5-Fr catheter as the sole instrument of drainage provides sufficient protective measure in pediatric pyeloplasty and also prevents leakage.

\section{Materials and Methods}

In a prospective clinical trial study, we performed 148 open pyeloplasties on 142 infants and children at the Bahrami Pediatric Hospital (Tehran University of Medical Sciences) over a seven-year period from 2005 - 2012. All cases in this study involved primary repair, although some were recurrent cases, and others had malrotated and/or horseshoe kidneys. We excluded patients with a single kidney (three cases) and some difficult recurrent cases (seven cases) from our study population. The exclusion was intended to provide more protective measures to prevent leakage and its consequences. In these excluded cases, we 
placed a perinephric Penrose drain in addition to an NU catheter.

All patients underwent preoperative renal ultrasound, nuclear diuretic renography (DTPA), and a DMSA renal scan with the measurement of differential renal function (DRF) and VCUG. The degree of hydronephrosis in our patients was classified in accordance with the society of fetal urology (USA) scheme. All parents were informed about the operative procedure prior to surgery.

Open pyeloplasty was performed via a $2.5-3.5 \mathrm{~cm}$ transverse posterior lumbotomy incision using a modified Anderson-Hynes technique. The modification involved the creation of a triangular flap from the lowest portion of the renal pelvis for performing an anastomosis to the laterally spatulated healthy proximal ureter with a length of $15-18 \mathrm{~mm}$. The abnormal stenotic segment of the ureter was then removed. Here, our intention was to create a funnel-shaped ureteropelvic junction (UPJ). We then made multiple side holes $10 \mathrm{~mm}$ apart in the $6-7 \mathrm{~cm}$ terminal portion of the 5-Fr catheter. The posterior layer of the pelvis flap was anastomosed to the spatulated ureter in the first step using 6 - 0 polyglactin sutures in a separate fashion. The catheter was then pulled through from the 2-mm skin stab wound with a right angle clamp into the Gerota fascia. The fine right angle clamp was placed into a lower calyx and then passed out through the parenchyma to grasp the catheter and pull it through into the pelvis. Then the catheter was sutured to the renal capsule with 4 - 0 chromic in a purse-string fashion to prevent postoperative dislodgement, and the catheter was placed across the anastomosis and down to the mid ureter. We emphasize the proper and careful placement of this catheter to ensure that at least two holes stay within the pelvis to allow the largest volume of urine to drain externally as well as the easy passage of urine down to the ureter. The repair was completed with the suturing of the anterior layer. The catheter was further secured to the skin at two points with nylon sutures, looped under the dressing, and then set to gravity drainage (Figure 1).

Postoperatively, the catheter was flushed every two hours with $2 \mathrm{~mL}$ of normal saline for 48 hours in the hospital in order to prevent blockage by blood clots. In addition, parenteral antibiotic therapy was administered for 48 hours. All patients were discharged two days postoperatively and were instructed to uncap the catheter and leave it to drain for 10 days at home under closed and sterile conditions. At the time of discharge from the hospital, $3 \mathrm{mg} / \mathrm{kg}$ trimethoprim-sulfamethoxazole was administered as a prophylactic agent for children and $10 \mathrm{mg} / \mathrm{kg}$ cephalosporin for infants and then continued for six weeks in each case.

A contrast study was performed 12 days postopera-

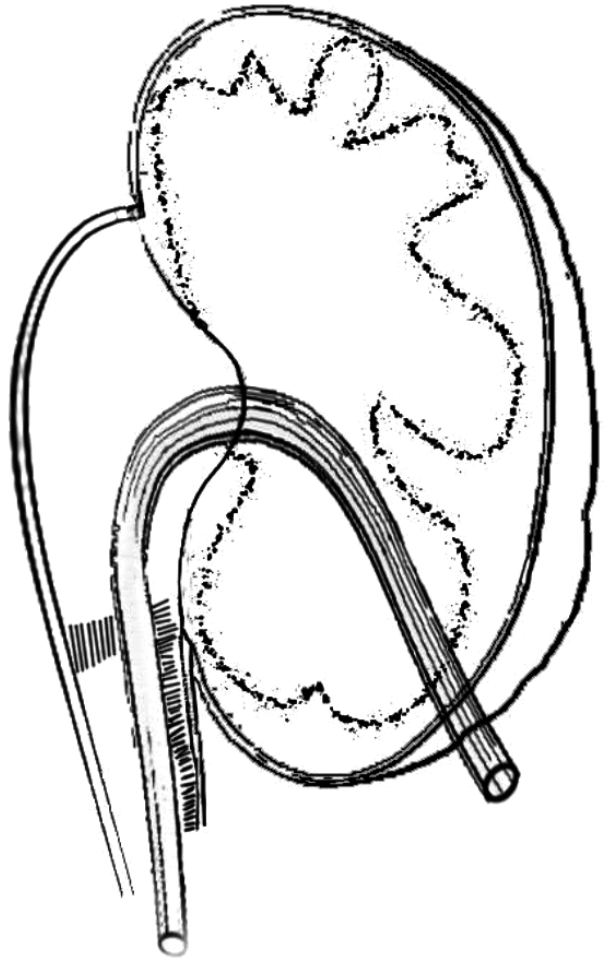

Figure 1. Schematic Diagram of a Nephroureteral Catheter and Funnel-Shaped Repair

tively, and the radiographic appearance was recorded. If no leak were identified, the catheter was removed in the clinic, which was the case for all patients. We routinely sampled the urine from both the catheter and the urethra for analysis and culture before the contrast study. Any complications that arose, such as poor catheter functioning, premature catheter dislodgement, urinary tract infection, blockage, and leakage around the catheter, were recorded.

\subsection{Ethics}

The study was approved by the research committee of the hospital affiliated with the Tehran University of Medical Sciences. It was also evaluated by an internal committee of pediatric surgeons.

\section{Results}

We performed 148 open pyeloplasties on 142 infants and children, including 6 with bilateral UPJ obstruction (UPJO) and 10 recurrent cases. Our study included 122 boys and 20 girls between the ages of 2 months and 11 years, and $76 \%$ of these patients were less than three years of 
age. We found that 118 (83\%) of our patients had grade-4 hydronephrosis, while 24 (17\%) were diagnosed with grade-3 hydronephrosis. In almost all of the patients, $\mathrm{t}_{1 / 2}$ was more than 20 minutes and varied between 20 and 38 minutes. The DRF in DMSA renal scanning ranged from $35 \%$ - 40\%. Table 1 shows the characteristics of the patients.

Table 1. Characteristics of the Patients

\begin{tabular}{|c|c|}
\hline Variables & Results \\
\hline Number of Pyeloplasties & 148 \\
\hline Number of patients (M/F) & $142(122 / 20)$ \\
\hline \multicolumn{2}{|l|}{$\%$ of UPJOs } \\
\hline Left & 70 \\
\hline Right & 26 \\
\hline Bilateral & 4 \\
\hline Number of associated VURs & 18 \\
\hline Number of UVJOs & 5 \\
\hline$\%$ Pelvic reduction & 48 (68 patients) \\
\hline Number of crossing vessels & 8 \\
\hline \multicolumn{2}{|l|}{$\%$ Presentation } \\
\hline Prenatal diagnosis & 64 \\
\hline Febrile UTI & 7 \\
\hline Pain & 16 \\
\hline Hematuria or trauma & 4 \\
\hline Incidental diagnosis or mass & 9 \\
\hline \multicolumn{2}{|l|}{ Age } \\
\hline$<6$, mo & 56 \\
\hline $6-18$, mo & 24 \\
\hline$>18 \leq 36, \mathrm{mo}$ & 28 \\
\hline$>3, y$ & 34 \\
\hline
\end{tabular}

${ }^{a}$ Values are expressed as \%.

Reduction pyeloplasty was performed in 68 patients (48\%). We encountered no significant difficulties during the intraoperative placement of this externalized NU 5-Fr catheter. No excessive bleeding occurred while passing the catheter through the renal parenchyma. Unintentional removal of the catheter did not occur in any of the patients when the catheter was in place and the patient was resting at home. In addition, no blockage or dislodgement of the catheter was noted.

A contrast study conducted through the catheter did not reveal any leakage across the repair 12 days postoperatively. Through this study, we were able to examine the whole length of the ureter all the way through to the bladder (Figure 2).
The catheter was removed on the day of the contrast study without requiring further anesthesia during an office visit. We did not encounter a symptomatic and/or febrile UTI during the 12 days while the catheter was still in place. The parents did not report encountering any difficulties at home, even with small children. The contrast study administered through a catheter in 148 pyeloplasty procedures identified 5 cases of UVJO, which were then individually and appropriately treated.

In this study, symptomatic UTI and transient obstructive symptoms occurred in about $10 \%$ of participants ( 15 cases) after stent removal during the following 1 - 3 days. This was the only significant complication in our study, and it was managed medically without any invasive procedures. All patients under 3 years of age were admitted to the hospital and were treated with intravenous antibiotics and hydration. Older patients were managed as outpatients. Patients who developed febrile UTI after the stent removal had no unique findings during the postoperative contrast study. In all of these patients, symptoms of febrile UTI and transient obstructive symptoms subsided after 2 - 3 days, and all except two remained unobstructed on long-term follow-up. A renal ultrasound scan was obtained six weeks after the pyeloplasty to ensure that the hydronephrosis (pyelocaliectasis) was improving. The diuretic renal scintigraphy (DRS) and DMSA scanning for measurement of the DRF was performed six months after the operation to provide a relative assessment of the overall renal function and washout time.

Improvement in the $t_{1 / 2}$ after pyeloplasty varied greatly. In some patients, the $t_{1 / 2}$ returned to the non-obstructed range in the first six months postoperatively. In others, this outcome took much longer. In many cases, the $\mathrm{t}_{1 / 2}$ remained in the equivocal range for at least six months. Of course, the renal function remained stable during this interval, and there was no need for concern. A nuclear study conducted one year postoperatively revealed normalization of $t_{1 / 2}$ in 146 pyeloplasties (8 - 13 minutes). Long-term imaging at three years was obtained to look for the delayed cicatrization and re-stenosis of the UPJ. Success was defined as improvement in hydronephrosis and stabilization or improvement in DRF function on DMSA renal scanning along with the normalization of the washout time on the DTPA diuretic renal scan (DRS).

In two patients (one bilateral, the other with unilateral UPJO associated with a giant abdominal mass and seizure disorder), cutaneous pyelostomy was performed as a primary procedure. The first pyeloplasty was performed six months later; after catheter removal, UTI occurred within two days, took a longer amount of time to resolve, and was managed only with considerable difficulty. In the follow-up evaluation, both patients demonstrated 

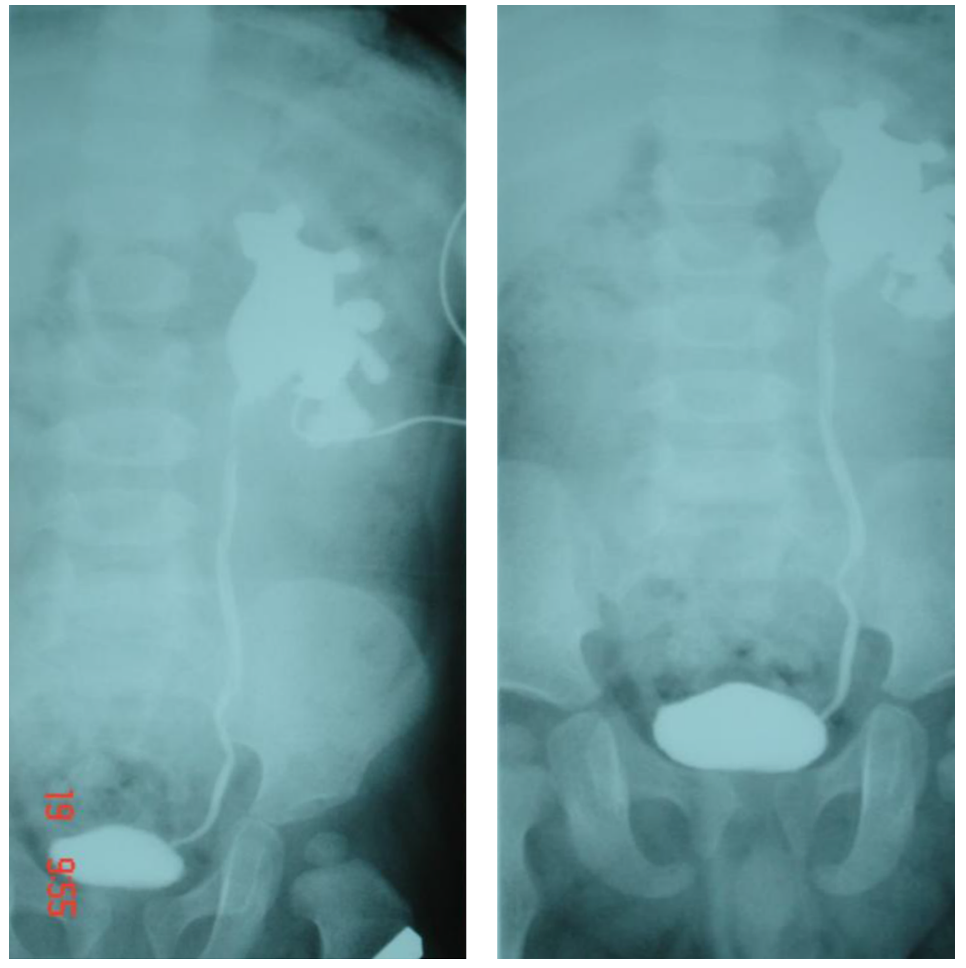

Figure 2. Contrast Study 12 Days Postoperatively Showing a Funnel-Shaped UPJ

increased washout times. We then performed repeat pyeloplasty in both cases. Intraoperatively, the UPJ seemed to be patent but was associated with a large, thick-walled pelvis. We assumed that in these two patients, the post-stent removal UTI had led to scarring of the anastomosis, although the large, thick-walled pelvis could have interfered with the washout across the anastomosis.

\section{Discussion}

The success rate of open pyeloplasty reported in the published pediatric literature is well in excess of 95\% (5). The original description of dismembered pyeloplasty advocates for non-stented repair, but as techniques have improved and as more postoperative complications were reported, drainage with stents and nephrostomy tubes have been used more liberally. However, postoperative drainage has not been uniformly reported in many published articles (6). Some authors have recommended a nephrostomy tube either with or without a stent to divert the urine and to keep the anastomosis dry (1). There is general agreement that the upper urinary tract should be drained after pyeloplasty in high-risk patients, such as those with poor renal function, extreme pyelocaliectasis, a single kidney, an inflamed renal pelvis, or a revision pyeloplasty (7). Different types of externalized and/or internal stents have been described (8). In addition, perinephric drains and urinary bladder catheters are used in a considerable number of cases.

The three most common reasons to use a stent and a nephrostomy tube are to ensure urinary diversion, to retain the ureteral caliber, and to maintain anastomotic patency and alignment. The main objective of draining the pelvis after pyeloplasty is to prevent urine leakage through the anastomosis. In addition, postoperative edema and undesirable kinking at the anastomosis site may cause ureteral occlusion that could lead to immediate and prolonged complications and hospitalization. These complications are worrisome to the surgeon as well as to parents $(1,9)$; stenting prevents these complications.

As reported in the literature, urologic complications may occur in both stented and non-stented patients, although they are slightly more frequent in non-stented groups (14\% vs. $12 \%$, respectively) $(4,10-12)$.

The NU catheter used in this study allows for maximal drainage of the pelvis, preventing any hydronephrosis secondary to edema of the anastomosis and also any early trans-anastomotic leakage and subsequent perianastomotic scarring. This NU catheter also provides for 
better alignment of the renal pelvis and ureter and facilitates external access to visualize the reconstructed area radiologically. This catheter remains in the renal collecting system for only 12 days postoperatively, whereas according to the published literature, routine operations involve a combination of devices, including a nephrostomy tube and a double pigtail ureteral stent, while a Penrose drain Foley catheter is used as a protective mechanism for 14 days, 2 - 6 weeks, 7-10 days, and 24 - 48 hours, respectively (13).

DJ stenting may be difficult or impossible in infants because of the small size of the UVJ (14). In contrast, the NU catheter diverts the upper tract only and does not cross the UVJ.

The only notable complication in our series was the occurrence of UTI in 15 of our 142 patients (148 pyeloplasties). In our opinion, the signs and symptoms of UTI and transient obstructive symptoms after removal of the stent may indicate anastomotic edema and malfunction, which can take several weeks to resolve, or as a complication of a foreign body (NU catheter) in the urinary tract (debris). We believe that the existence of debris in the stented urinary tract and its associated transient obstructive effects in the ureter may play a role in this complication. In most of these cases, we found that the urine samples obtained through the external catheter before its removal usually contained a number of white blood cells, red blood cells, and bacteria. Although the fever and positive urine culture seen in patients with DJ can be due to reflux (14), we have been unable to determine why some patients develop UTIs after catheter removal while others do not. More work is needed in this area to prevent UTIs and identify their causes.

Two of the most important concerns of an externalized drainage tube are its poor functioning and dislodgement before its intended removal. When this technique was implemented in our clinical trial, we did not observe any premature dislodgment or unintentional removal. As previously mentioned, this catheter was the sole method of drainage in our patients. We emphasize proper placement and securing of the catheter intraoperatively with the aim of keeping it in a fixed position within the renal pelvis for the desired length of time.

Many reports in the literature indicate that external drainage techniques are unequivocally associated with longer hospital stays. In our study, all patients were discharged 48 hours postoperatively. However, in a comparative study, although there was a longer stay for patients with a nephrostomy tube, it had lower overall costs (14). The 48-hour hospital stay was intended to provide acute care management, primarily due to the need for intravenous hydration, antibiotic therapy, parenteral pain con- trol, flushing of the catheter to prevent blockage, and educating parents in caring for the external drainage catheter.

Another problem is the inconvenience of having an external drainage device for the child and his or her parents. of course, the problem is varied in different cultural and social environments. In the opinion of some authors with whom we have had personal communication, performing a contrast study through the tube that crosses the UPJ is not very useful in demonstrating leaks in the suture line, nor does it demonstrate patency of the anastomosis that is being stented by the tube. However, this procedure can actually show distal patency.

One other drawback to an externalized ureteral stent as reported in the literature is the increased risk of UTI (as seen in 15 of our patients), a prolonged hospital stay, and restricted mobility of the patient postoperatively (12). The creation of local ischemia, pressure necrosis, and subsequent stricture formation, particularly in the small caliber ureters, has also been attributed to the presence of a ureteral stent (12). We did not experience these complications in any of our patients.

One clear and main limitation of this study is the lack of a comparative control group. Although a historical control group is not sufficient, in the past we would have applied some additional measures to better ensure appropriate drainage, including a nephrostomy tube in addition to the ureteral stent, perinephric drains, and a Foley catheter. With experience, we decided that a single NU 5-Fr catheter as the sole method of drainage without any additional catheter or drain usually addresses the required protection for pyeloplasty. Therefore, we set up this descriptive case series using only a NU 5-Fr catheter for the pyeloplasty.

This method has not been widely used and also carries the message that in children, a single 5-Fr catheter is sufficient for the prevention of postoperative leakage. There is no fear of dislodgement and migration or mechanical irritation of the bladder trigone, which are often seen with DJ catheters. While these conditions might also occur with NU catheters that have been used for quite some time, they are often associated with the concurrent use of some other protective measures, such as a Foley catheter or perinephric drain.

In pediatric pyeloplasty for the correction of congenital UPJO, radiologic exploration of the distal ureter is critical. Many centers employ preoperative cystoscopy and retrograde ureterography. In our patients, we performed this task with antegrade contrast study through the catheter and found five cases of UVJO. We treated this second anomaly individually. 


\subsection{Conclusions}

The use of a percutaneous externalized 5-Fr NU (feeding tube) with multiple side holes as the sole instrument for diversion after open pyeloplasty was sufficient to prevent anastomotic leakage. Patent anastomosis was achieved in $98.7 \%$ of the cases according to long-term follow-up, and there were only a few notable complications. The catheter was well tolerated and offered the combined advantages of a nephrostomy tube and an internal stent and also allowed for radiologic study of the anastomosis and the distal ureter while obviating the need for a second anesthetic for its removal. Therefore, it can be a good option during pyeloplasty, especially in developing countries with low economies and poor medical facilities.

Further research is required to identify ways to prevent the risk of symptomatic UTI associated with an externalized NU catheter after its removal.

\section{References}

1. Austin PF, Cain MP, Rink RC. Nephrostomy tube drainage with pyeloplasty: is it necessarily a bad choice?. J Urol. 2000;163(5):1528-30. [PubMed: 10751882].

2. Arda IS, Oguzkurt P, Sevmis S. Transanastomotic stents for dismembered pyeloplasty in children. Pediatr Surg Int. 2002;18(2-3):115-8. doi: 10.1007/s003830100660. [PubMed: 11956775].

3. Mandhani A, Goel S, Bhandari M. Is antegrade stenting superior to retrograde stenting in laparoscopic pyeloplasty?. J Urol. 2004;171(4):1440-2. doi: 10.1097/01.ju.0000116546.06765.d1. [PubMed: 15017193].
4. Smith KE, Holmes N, Lieb JI, Mandell J, Baskin LS, Kogan BA, et al. Stented versus nonstented pediatric pyeloplasty: a modern series and review of the literature. J Urol. 2002;168(3):1127-30. doi: 10.1097/01.ju.0000026415.22233.d7. [PubMed: 12187251].

5. Braga LH, Lorenzo AJ, Farhat WA, Bagli DJ, Khoury AE, Pippi Salle JL. Outcome analysis and cost comparison between externalized pyeloureteral and standard stents in 470 consecutive open pyeloplasties. J Urol. 2008;180(4 Suppl):1693-8. doi: 10.1016/j.juro.2008.05.084. [PubMed: 18708220] discussion1698-9.

6. Baniel J, Livne PM, Savir A, Gillon G, Servadio C. Dismembered pyeloplasty in children with and without stents. Eur Urol. 1996;30(3):400-2. [PubMed: 8931977].

7. Carr MC. In: The Kelalis-King-Belman Textbook of Clinical Pediatric Urology. Docimo SG, editor. UK: Informa Healthcare Ltd; 2007. pp. 479-86.Ureteropelvic junction obstruction and multicystic dysplastic kidney: surgical management.

8. Zaidi Z, Mouriquand PD. The use of a multipurpose stent in children. BrJUrol. 1997;80(5):802-5. [PubMed: 9393307].

9. McMullin N, Khor T, King P. Internal ureteric stenting following pyeloplasty reduces length of hospital stay in children. $\mathrm{Br} J$ Urol. 1993;72(3):370-2. [PubMed: 8220999].

10. Yucel S, Samuelson ML, Nguyen MT, Baker LA. Usefulness of short-term retrievable ureteral stent in pediatric laparoscopic pyeloplasty. J Urol. 2007;177(2):720-5. doi: 10.1016/j.juro.2006.10.017. [PubMed: 17222666] discussion 725

11. Hussain S, Frank JD. Complications and length of hospital stay following stented and unstented paediatric pyeloplasties. Br J Urol. 1994;73(1):87-9. [PubMed: 8298904].

12. Ahmed S, Crankson S. Non-intubated pyeloplasty for pelviureteric junction obstruction in children. Pediatr Surg Int. 1997;12(5-6):389-92. [PubMed: 9244107].

13. Olsen LH, Rawashdeh YFH. In: Campbell-Walsh Urology. Wein AJ, editor. Philadelphia: Elsevier; 2016. pp. 3057-75.Surgery of the Ureter in Children.

14. Garg RK, Menon P, Narasimha Rao KL, Arora S, Batra YK. Pyeloplasty for hydronephrosis: Issues of double J stent versus nephrostomy tube as drainage technique. J Indian Assoc Pediatr Surg. 2015;20(1):32-6. doi: 10.4103/0971-9261.145444. [PubMed: 25552829]. 